\title{
Reflection on the Creative Design of MOOC's Micro Video based on Visual Culture
}

\author{
Han Liu ${ }^{1, a}$, Yongjun Guan ${ }^{2, b}$ and Min $\mathrm{Qu}^{2, c}$ \\ ${ }^{1}$ School of Communication and Artistic Design, Wuhan Donghu University, Wuhan 430000, China; \\ ${ }^{2}$ School of Finance \& Economics, Guangxi University of Science and Technology, Liuzhou 545000, \\ China. \\ alh409231@qq.com, b30670995@qq.com, '35824663@qq.com
}

Keywords: Creative design, Micro video, visual culture theory.

\begin{abstract}
This article explains and demonstrates how to design MOOC's micro video creatively, from the perspective of the theory of visual culture. The authors first discuss the influence of visual psychology on video design, then put forward some creative design thinking on the scene, the character and the courseware, finally give the conclusion that the real good idea is the organic integration of these three elements, which can create a stylish and high quality video through using the modern information processing technology and video editing software.
\end{abstract}

\section{Introduction}

Micro video as a form of media has been widely used in education, especially with the development of the concept of MOOC in recent years. The role of micro video in teaching is becoming more and more prominent. MOOC is the abbreviation of Massive Open Online Courses, which is a vitual university without walls, providing a large-scale open network courses. In order to attract the attention of students, it has to rely on its own core content. And the main form of this teaching method is micro video, which must be a "work of art", reflecting a teacher's wisdom and teaching philosophy. A form of creative expression is really reguired throughout its design and production, and only in this way can truly break the time, geographical restrictions, and attract the most extensive attention of students. This article will analyze the possibility of the creativity from the perspective of the theory of visual culture .

\section{The inspiration of visual culture theory to the creative design of micro video}

The cultural patterns, such as images, symbols, animation and other visual elements, which can be directly obtained and interpreted by visual perception, are regarded as the form of visual culture. [1] In the process of human cognition to the outside world, there are about more than $80 \%$ of the information is obtained by visual senses. Visual properties has become the dominant factor of culture, and the extensive visualization has profoundly changed the form of many cultural activities, showing a tendency of picture overwhelming text. With the progress of visual technology extending the human's visual perception continuously, People's visibility requirements also continue to rise quickly. At the same time, the virtual technology make a greater performance space for visual arts, new visual patterns are cropping up endlessly.

From the perspective of visual psychology, people's perception system has a tendency to combine various things into simple units. In the design of micro video, we should consider the reasonable distribution of visual elements, so that the screen has a clear visual focus and reasonable context. Excellent micro video learning resources must be the perfect form to express content. We should pay attention to these visual factors as follows in the design of micro video. 


\section{1. the unity of whole and part}

The sense of wholeness is the proper connection between the visual elements of a picture. [2] In the design of micro video, the integral feeling relates to two levels of organic connection, one of which refers to all the visual elements within the picture, another of which involves a single picture and the whole video. Micro video should be focus on overall design and layout. For example, the beginning and end of the video, the screen background, the font and the color arrangements, etc. should be used as a system of planning, in order to make each part obedience to the whole while also changeable. The micro video should form a complete framework, and design different function modules in the overall structure. This not only ensures the diversity of the local form, but also creates a harmonious, beautiful and vivid micro video as a whole, which gives the learners a overall sense of coordination. Video, audio, animation, images, text and other multimedia information elements, are all the important content of micro video's design. The series of elements should be co-ordinated and planned reasonably at the early stage of the film shooting, make their structure and color unified to overall personality style.

\section{2. the contrast and harmony of color}

Color design includes the main colors of the interface as well as the color of the text. Select the appropriate interface colors according to the learning content and the characteristics of the learners, and pay attention to the contrast and harmony of color. Generally speaking, the background should be dark, in order to reduce the interference to learners, while the color of the content should be more bright for attracting attention. Common collocation are black and white, blue and white, etc. In addition, the categories of the picture color should not be too many, otherwise it is easy to increase the learner's external cognitive load and prolong reaction time. If long time study, learners can easily cause visual fatigue. [3]

\section{3. a balance between dynamic and static}

The visual elements used in the form of dynamic or static performance should be fully considered in the design of the video. Add special motion effects to some visual elements, there will be a good result with soothing music. When the voice mentioned something, the scene changes to the corresponding pictures of the things which appear dynamic, and add soft music not affecting main sound, so that you can alleviate the pressure and gain the joyful learning experience as a learner, because of the colorful video style.

\section{Creative design method for MOOC's micro video}

The design of MOOC's Micro Video based on Visual Culture means combining the appropriate teaching content and the tailor-made picture. The picture's design must have outstanding theme, clear content, visual aesthetics, so that this design of the interface, not only increase the visual effect, but also can fully mobilize students' learning interest and improve teaching effect. Through the combination of a variety of visual elements, we can make creative design from these aspects.

\section{1. creative design of the scene}

Micro video scenes mainly include real, studio and virtual scene. In the real scene case, the scene design has been more and more diversified. Pictures used to shoot mainly in the classroom, laboratory, office, etc. now break through the narrow sense of "learning" environment, starting to capture the nature scene, or make all kinds of cultural landscape as the background. A teacher can walk in the scenic spots and talk brilliantly. Gardening taught is more convenient in the nursery, providing a lively scene. Some case of scenario can be restored to the reality by shooting in the event site or processing raw materials. For those is not easy to shoot or have special difficulties, we can use digital $3 \mathrm{D}$ technology to design a virtual scene, then merge the characters and other items into it through the Key Effects. To sum up, the teaching scene in micro video has changed greatly, and this change is consistent with the goal of teaching method reform. This form just make up for the inadequacy of 
scene elements in realistic teaching, with introducing a relaxed and pleasant situation into teaching, will greatly improve the intuitive and sensibility of teaching content. In practice, the scene elements are also flexible which can take a combination form of multi scene and studio.

It should be noted that the selections of the scenes not only those learners are interested in, but also related to the teaching content. During the period of sound recording, it is better to use the high frequency characteristic, high resolution, and high sensitivity of capacitive microphone. Because the live action are greatly influenced by the environmental noise, it is necessary to denoise in the editing. In the case of studio shooting, the background should be simple and clear, the theme should be matched to the course, the color should not be too bright, and the scene can also add some small ornaments to regulate the atmosphere.

2. creative design of the character

The characters in the micro video course are mainly teachers, followed by the students and the characters in the case situation. Teacher's image in front of the camera is essential to the visual effect of the video. The teachers' appearances, behaviors, expressions, operations, etc. are also a range of visual culture elements, which deserve meticulous preparation before the shooting. The teacher should be full of spirit, make clear speech; grooming and generous; natural looking and cordial[4]. Sometimes, teaching is a performing arts with image, emotion, and experience, requiring teachers to master the art of the stage as well as the actors, who can use of hand, eye, body, method and step properly. In the teaching performance, teachers have strong expressive force, appeal and attraction, which can activate the knowledge, optimize the learning process; wake up emotions, creating learning environment; start the emotion, enhance learning motivation; stimulate interest, show the charm of learning, and eventually make learning become a kind of happiness, not a worry thing.

On the other hand, if we can change the camera lens to highlight the main characters in the picture when filming, the picture will be lively and rich layering. A host camera is used to record most of the panoramic view of the classroom and teaching process, generally giving the speaker's front view; another camera is placed at the front side of the platform to the 45 degree angle, which is used to capture the state of students' listening and teaching activities' features. In order to make teachers appear modest on the screen, camera height can be slightly lower than the sight line of the teacher, using close shot or medium shot. In order to enhance the study effect of learners and prevent visual fatigue, you'd better use the pushing, pulling, Shaking, moving techniques properly. It means recording the teaching activities objectively and accurately by different shot size. Take every shot up and down frame of the picture rigorously, and pay attention to the main body's position in the frame structure. In final editing, you can increase the movement of characters and pictures by moving the axis, and arouse the audience's attention by dynamic visual stimulation.

In a word, teacher is the most important actor in MOOC's micro video. Teachers' speech ability and moderate performance ability in front of the camera, which means mastering the language of television, staying aplomb, and combined with a generous, decent body language, can increase the visual aesthetic feeling of the picture.

\section{3. creative design of the courseware}

According to the theory of visual culture, the text symbols are abstract, universal, which express the deep level of concept to be easily understood by some people; image symbols have intuitive, similar and other characteristics, through which the readers will have an immersive feeling. On the communication effect, the text symbols are not fresh enough, which need the audience to savor words of artistic conception carefully what the author wants to convey. Image symbols, however, are intuitive, and the information spreads rapidly and directly. "What you see is what you get": when people see the germinal grass, they understand that the spring is coming. They are more intuitive than the words. 
In the traditional courseware design, texts are often more than the images. In the continuous improvement process, charts, diagrams, and even animations began to gradually introduced in the courseware. For example, set a dynamic cartoon image in the lower right corner of the courseware, which can not only enrich the courseware content, but also increase the dynamic of the picture, so as to mobilize the attention of the viewers. With the development of the production technology, a single graphics or animation has been unable to meet the demands of the audience's visual improvement, which require a more comprehensive design of the courseware. A very good example: there are two kinds of stylish design in Microlecture • China ${ }^{1}$. One called "dynamic courseware", which means more than just the dynamics of elements used in courseware, such as beating characters or dynamic chart. The more important is, the whole effect of the courseware is a three dimensional space, the conversion between courseware pages is in a virtual gallery space by shaking shot, the courseware itself has become a scene of a micro video. Another is called "rich material", the design is a more flexible processing and fusion of a variety of diverse material. If the teacher says "coast", the video presents the real coastline images, and explains with dynamic text or graphics subsequently. Sometimes the teaching picture is fused together, through the split or blend of the picture, forming a rich form. The creativity of this courseware has already broken the independence of itself. In other words, scene, character and courseware have been completely integrated, regardless of each other.

\section{Conclusion}

MOOC's micro Video is an effective form of learning, of which the short style with the concise content, are very consistent with the current people's habit of watching. How to make full use of the information technology and professional teaching, so as to give more richly creative and visual performance of the teaching videos, and convey the teaching content and good teaching style more effectively, has become the key issue for MOOC's further development. Visual culture theory can provide a creative thinking professionally, and ensure to develop a high quality of micro video, which is planned carefully, recorded thoughtfully and edited professionally.

\section{Acknowledgments}

This article is funded by The Humanities and Social Science Project of the Education Department of Hubei Province in 2016: The study on video communication strategy of Jingchu Tourism Culture in the "Internet+" era. The authors also wish to acknowledge the support of the Teaching and Research Project of Wuhan Donghu University.

\section{References}

[1]Shuyu zhang. An introduction to visual culture [M]. Nanjing: jiangsu people's publishing house, 2003:7-14.

[2]Wenxiang Qiu, Huiyin Zhan. Research on the application of the theory in the design of network course[J]. China Educational Technology, 2006(9):82-83.

[3]Chi Chen. Research into the Interface Design of Web-based Cours [D]. Shanghai International Studies University, 2012:11-12.

[4]Yan Tang, Nianchun Wang. The specialization strategy of network course video production based on the visual culture thinking[J]. Software Guide • Educational Technology, 2011, 4 (7) : 69-71.

\footnotetext{
${ }^{1}$ Information on: http://www.vkzg.cn/.
} 